\title{
REMARQUES SUR LE CANON DES DÉFUNTS ÉCRIT PAR S. CASSIA DE CONSTANTINOPLE
}

Parmi les œuvres hymnographiques de la célèbre poétesse byzantine S. Cassia qui nous sont parvenues il y a un canon des défunts. Il a été édité par K. Krumbacher d'après le manuscrit Cod. Crypt. $\Gamma . \beta . \mathrm{V}$ fol. $1^{\mathrm{v}}-6,{ }^{1}$ et traduit en anglais par A. Tripolitis ; ${ }^{2}$ récemment, j'ai fait une traduction du canon en slavon et en russe, avec des commentaires. $^{3}$

Je voudrais ici faire quelques remarques sur les théotokions de ce canon. Ces tropaires ne s'inscrivent pas dans l'acrostiche et ne sont pas originales : Cassia les a pris tous, à l'exception du théotokion de la $9^{\text {ème }}$ ode, des canons différents qui se trouvent même à présent dans les livres liturques. I. Rochow l'a déjà noté mais elle a estimé faussement que le théotokion de la $5^{\text {ème }}$ ode est né sous la plume de Cassia ; ${ }^{4}$ en fait il coïncide presque entièrement avec celui de la $5^{\text {ème }}$ ode du $2^{\text {ème }}$ canon (du $8^{\text {ème }}$ ton, ainsi que le canon écrit par Cassia) à l'archistratège Michel, le 8 novembre aux matines. Comparons : ${ }^{5}$

(1) K. Krumbacher, Kasia, Sitzungsberichte der philosophisch-philologischen und der historischen Classe der k.b. Academie der Wissenschaften (1887) Heft. 1. 347-356. Ici je cite le canon d'après cette édition.

(2) A. Tripolitis, Kassia: the Legend, the Woman, and her Work (New York-London, 1992) (Garland Library of Medieval Literature. Serie A, 84) 88-105.

(3) Монахиня Кассия (Т. А. СЕнинА), Канон об упокоении усопших, творение св. Кассии Константиноподьской, dans : История и теория культуры в вузовском образовании, вып. 4 (Новосибирск, 2008) 211-226.

(4) I. Rochow, Studien zu der Person, den Werken und dem Nachleben der Dichterin Kassia (Berlin, 1967) (Berliner Byzantinische Arbeiten, 36).

(5) Les divergences sont en italique. 


\section{Canon des défunts}

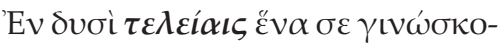

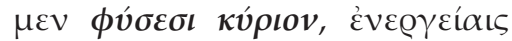

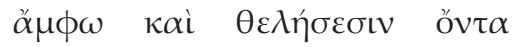

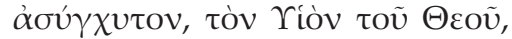

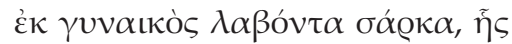

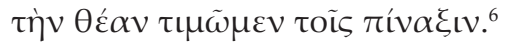

\section{Canon à l'archistratège Michel}

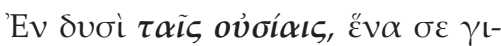

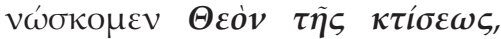

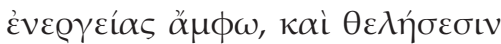

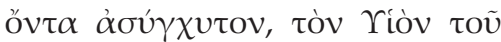

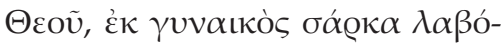

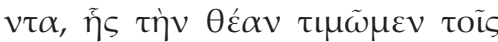
$\pi i ́ v \alpha \xi ı .^{7}$

Les ménées grec et slavon indiquent que le canon à l'archistratège Michel a été écrit par le moine Jean, ${ }^{8}$ mais l'acrostiche de ses théotokions donne le nom $\mathrm{K} \lambda \eta \dot{\eta} \mu \varepsilon v \tau o \zeta$, ce qui est noté dans le ménée grec et n'est pas noté dans le ménée slavon. En réalité, ce canon est sorti tout entier de la plume de $S$. Clément l'Hymnographe qui vivait à l'époque des deux périodes de l'iconoclasme. ${ }^{9}$ Le canon contient des mentions de la veneration des icônes (par exemple, les théotokia des odes 4, 6 et 7), de «l'union des églises et la chûte de toute hérésie résistante » (ode 1, tropaire 3). A. Kazhdan a montré que le canon des archanges avait été écrit par $\mathrm{S}$. Clément, évidemment après la réstauration des icônes au VII Concile œcuménique. ${ }^{10}$ Cet emprunt du tropaire n'a été remarqué ni par I. Rochow, ni par A. Tripolitis, car celle-ci suit en tout celle-là, et Rochow s'appuie sur E. Follieri qui cite les tropaires de S. Clément et de S. Cassia comme des œuvre différentes. ${ }^{11} \mathrm{~N}$. Tsironis a bien fait attention à ce théotokion, à son contenu christologique et à la mention directe des icônes, mais elle ne s'est pas aperçue non plus de la parallèle avec le théotokion de $\mathrm{S}$. Clément. ${ }^{12}$

(6) Krumbacher, Kasia..., 351, 1. 110-116.

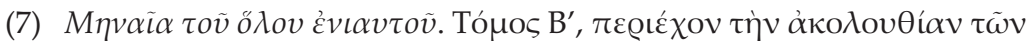

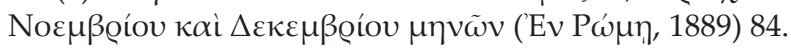

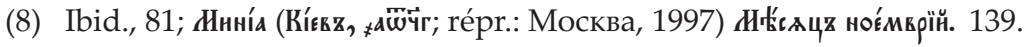

(9) Cf. A. P. Kazhdan, A history of Byzantine literature (650-850) (Athens, 1999) Pt. 2, ch. 5, avec la bibliographie ; je cite cet ouvrage dans la traduction russe : А. П. КАЖдАН, История византийской литературы (650-850 г2.). Пер. с англ. А. А. Белозеровой и др. (Санкт-Петербург, 2002) (Византийская библиотека: исследования) 337-348.

(10) КАЖдАН, История византийской литературы..., 338-339.

(11) Cf. H. Follieri, Initia hymnorum ecclesiae graecae, vol. 1 (Vatican, 1960) (Studi e Testi, 211) 437.

(12) N. Tsironis, The Body and the Senses in the Work of Cassia the Hymnographer : literaty Trends in the Iconoclastic Period, $\Sigma \Upsilon M M E I K T A 16$ (2003) 151. 
Apparemment, S. Cassia a emprunté ce théotokion comme significatif dans le contexte de la polémique anti-iconoclaste et accentue un

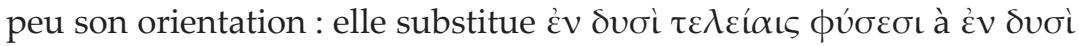

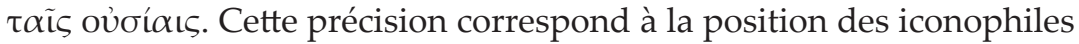
qui insistaient que si le Christ est devenu, dans son incarnation, l'homme parfait, il peut et doit être circonscriptible comme chaque homme, et ce sujet était discuté surtout pendant le second iconoclasme, justement à l'époque de S. Cassia : les iconoclastes traitaient les iconophiles comme néstoriens, et les iconophiles accusaient leurs adversaires de monophysisme parce que, d'après la doctrine iconoclaste, l'humanité du Christ est d'une nature commune, ses idiomes physiques sont accidentels, et la nature humaine du Christ est invisible. ${ }^{13}$

A. Kazhdan n'a pas fait attention à ce que les théotokia du canon ne font pas partie de l'acrostiche; en examinant le contenu du dernier théotokion, il fait une réserve : "Cassia, si elle est l'auteur de cette partie du canon (les theotokia, s'ils n'étaient pas "signés", pouvaient passer d'un auteur à l'autre, comme montre le cas de Clément), change subitement de sujet... ». ${ }^{14}$ Mais nous voyons, sur l'example de S. Cassia, que les hymnographes byzantins employaient et remaniaient les théotokia anonymes comme "signés".

$\mathrm{N}$. Tsironis pense que le théotokion cité « offers us a terminus post quem for the date the author composed the poem, which must have been after 843 , since such an open assertion of the veneration of the images could not have circulated prior to the Triumph of Orthodoxy ». ${ }^{15}$ Cet argument me semble assez faible. En premier lieu, Cassia écrivait ses œuvres hymnographiques avant tout pour chanter dans son propre monastère, ce que montre son fameux hirmos pour la 1 ère ode du canon du Grand Samedi écrit de la part des jeunes filles : $\dot{\alpha} \lambda \lambda^{\prime} \eta \dot{\eta} \mu \varepsilon \bar{\varsigma}$

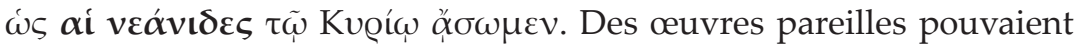
rester entre les murs du couvent pendant des années ou ne se répandre que dans un cercle d'intimes, et pour Cassia c'étaient sans doute les iconophiles. En deuxième lieu, attendre le Triomphe de l'Orthodoxie pour parler ouvertement de la vénération des images n'était pas

(13) Cf., par ex., B. Lourié, Le second iconoclasme en recherche de la vraie doctrine, SP 34 (2001) 150-153 ; sur la théologie des iconophiles et des iconoclastes en VIII-IX siècles cf. В. М. ЛурьЕ, В. А. БАРАНов, История византийской философии. Формативный период (Санкт-Петербург, 2006) 430-486, surtout 441, 471-485.

(14) КАЖДАН, История византийской литературью.., 411.

(15) Tsironis, The Body and the Senses in the Work of Cassia..., 151, n. 53. 
propre aux iconophiles ${ }^{16}$ et d'autant plus à S. Cassia qui a été même flagellée pour son iconophilie. On peut aussi se rappeler une de ses épigrammes qui montre bien le caractère de la poétesse : « Je hais celui qui se tait quand il est temps de parler $»{ }^{17}$ Toutefois, il y a d'autres raisons pour dater son canon d'un temps beaucoup postérieur à 843 , et j'en parlerai plus bas.

Je dois aussi noter qu'il est impossible de partager l'opinion de Tsironis que le théotokion de la $5^{\text {ème }}$ ode du canon de défunts « is perhaps the most direct reference to the veneration of image in the entire homiletic and hymnographical corpus of the middle Byzantine period $\gg .{ }^{18}$ On peut trouver beaucoup de mentions des icônes et même d'invectives contre les iconoclastes, par exemple, dans les œuvres d'un autre fameux hymnographe, contemporain de Cassia, S. Théophane le Graptos. Voici quelques citations :

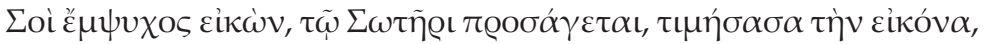

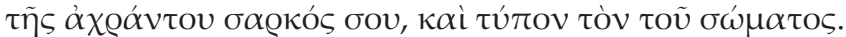

(Canon en l'honneur de S. Euthyme de Sardes, ${ }^{19}$ le 26 décembre, ode 5 , tropaire 1.)

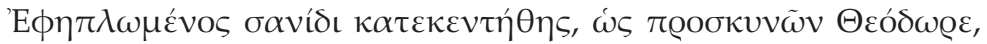

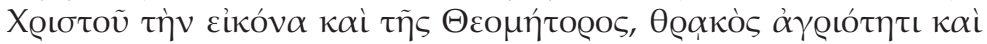

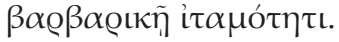

(Canon en l'honneur de S. Théodore le Graptos, ${ }^{20}$ le 27 décembre, ode 4 , tr. 1.)

(16) Par exemple, S. Théodore le Stoudite répétait constamment que les orthodoxes ne devaient pas garder le silence pendant les persécutions, même si l'empereur interdit de parles des icônes, parce que c'est une vraie trahison de la foi ; cf. G. Fatouros (rec.), Theodori Studitae epistulae, vol. II (Berlin-New York, 1992) (CFHB, 31) Ep. 149, 1. 1-46; 312, 1. 9-18; 425, 1. 10-32. Voir aussi plus bas quelques observations sur les canons écrits par $S$. Théophane le Graptos.

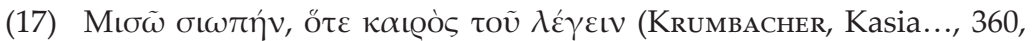
1. 90$)$.

(18) Tsironis, The Body and the Senses in the Work of Cassia..., 151.

(19) S. Euthyme a été martyrisé en 831, et S. Théophane a écrit ce canon avant le Triomphe de l'Orthodoxie, cf. pour les détails T. А. Сенина (монахиня Кассия), Несколько замечаний по поводу Жития св. Евфимия Сардского, Scr 2 (2006) 411-417. Dans ce canon l'empereur iconoclaste Léon l'Arménien

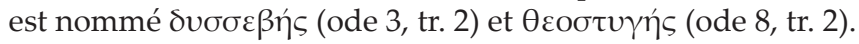

(20) Le frère de $S$. Théophane ayant subi les souffrances avec celui-ci sous l'empereur Théophile est mort en 841. La vénération des icônes est mentionnée aussi dans le $3^{\text {ème }}$ tropaire de l'ode 7 ; dans quelques tropaires il y a des invectives contre les empereurs iconoclastes ( $1^{\text {er }}$ et $3^{\text {ème }}$ de l'ode $3,1^{\text {er }}$ de l'ode 8$)$. 


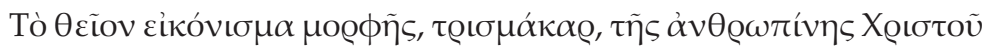

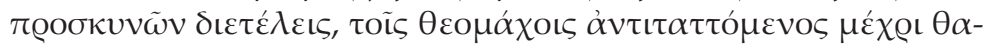

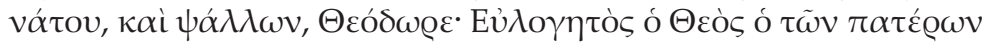
$\eta \dot{\eta} \mu \tilde{\omega} v$.

(Canon en l'honneur de S. Théodore le Stoudite, ${ }^{21}$ le 11 novembre, ode 7, tr. 2 . $)^{22}$

Ainsi, nous voyons que $\mathrm{S}$. Cassia n'était point la seule à faire la démonstration de ses convictions iconophiles dans les œuvres hymnographiques.

Dans le théotokion de la $9^{\text {ème }}$ ode, apparemment le seul de la plume de Cassia, la poétesse prie Dieu de " couronner l'empereur croyant » et $\mathrm{d}^{\prime}$ « exterminer la force des ennemis » :

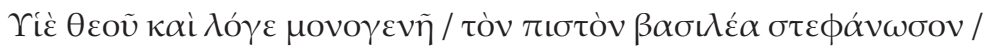

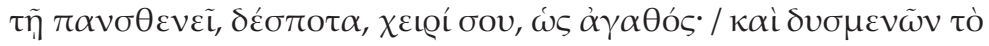

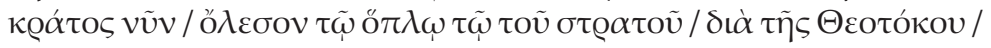

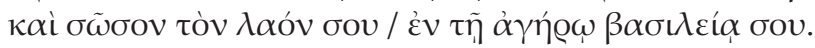

Évidemment il s'agit de la guerre avec les Arabes, et ó $\pi$ ı $\sigma$ tò $\beta \alpha-$ $\sigma \iota \lambda \varepsilon u ́ s$ ne peut être autre que Michel III. Peut-être Cassia a en vue les conflits des années $860^{\mathrm{e}}::^{23}$ le $3^{\mathrm{e}}$ tropaire de la dernière ode, où Cassia prie les «pères, frères, parents, amis et les unanimes » décédés de prier Dieu pour elle, montre que le canon a été écrit par la poétesse probablement à un âge avancé, peut-être vers 860 quand beaucoup de ses parents et amis ${ }^{24}$ était déjà morts et quand elle-même réfléchissait sur son passage à l'autre monde. Le théotokion de la $5^{\text {ème }}$ ode orienté contre les iconoclastes n'y change rien parce que la polémi-

(21) S. Théodore est mort en 826 ; cf. dans ce canon encore les mentions des images sacrées dans les odes 1 (tr. 3 ) et 8 (tr. 1).

(22) On peut multiplier les exemples. Je me propose de consacrer un article particulier aux sujets liés avec la lutte contre l'iconoclasme dans les œuvres de S. Théophane le Graptos.

(23) Pour les détails des guerres entre les Byzantins et les Arabes sous Michel III cf. А. А. ВАсильев, Византия и арабъ. Политические отношения Византии и арабов за время Аморийской династии (Санкт-Петербург, 1900) 153-210 ; sur les batailles des années soixante cf. p. 189-210. Cf. aussi H. GréGOIRE, Études sur le neuvième siècle, Byzantion 8 (1933) 515-538, sur les batailles de 853,863 et 866 .

(24) Parmi lequels était, par example, le fameux S. Théodore le Stoudite († 826), avec qui Cassia était en correspondance dans sa jeunesse ; sûrement, Cassia connaissait aussi ses disciples, y compris S. Naukratios († 848) et d'autres stoudites dont plusieurs étaient déjà morts vers 860 . 
que sur la veneration des images restait actuelle à Byzance longtemps après $843 . .^{25}$

\section{SUMMARY}

The article contains an analysis of the hymnographic canon for the deaths written by Kassia of Constantinople. It is shown that all its theotokia (apart from the last one) are taken from earlier canons, including the theotokion of the fifth ode that was previously considered as written by Kassia herself. Concluding from its contents, the canon is to be dated to the 860s.

(25) Même jusqu'à la période du patriarcat de S. Photios; cf., par exemple, Ф. М. Россейкин, Первое правление Фотия, патриарха Константинопольского (Сергиев Посад, 1915) 143, 474-475; А. П. АЕБЕдев, История Константинопольских соборов IX века (Санкт-Петербург, ${ }^{2} 2001$ [Москва, $\left.{ }^{1} 1888\right]$ ) (Византийская библиотека: исследования) 166-169 ;F. DvorNIк, The Patriarch Photius and Iconoclasm, DOP 7 (1953) 69-97 ; J. Goulllard, Deux figures mal connues du second iconoclasme, Byzantion 31.2 (1961) 387-401 ; C. Mango, The Liquidation of Iconoclasm and the Patriarch Photios, dans: A. Bryer, J. Herrin (eds.), Iconoclasm. Papers given at the Ninth Spring Symposium of Byzantine Studies. University of Birmingham. March 1975 (Birmingham, 1977) 133-140 ; D. Stratoudaki-White, Patriarch Photios and the Conclusion of Iconoclasm, GOTR 44 (1999) 341-355. 